\title{
Determinaciones de niveles de creatina y lípidos mediante espectroscopia por resonancia magnética en miocardio de pacientes con insuficiencia cardiaca no isquémica
}

\author{
JOSÉ LUIS WINTER ${ }^{1}$, PABLO CASTRO ${ }^{1}$, LUIS MENESES ${ }^{2}$, MÓNICA CHALHUB ${ }^{1}$, \\ HUGO VERDEJO $^{1}$, DOUGLAS GREIG ${ }^{1}$, LUIGI GABRIELLI', \\ MARIO CHIONG ${ }^{3}$, ROBERTO CONCEPCIÓN ${ }^{4}$, ROSEMARIE MELLADO ${ }^{5}$, \\ CLAUDIA HERNÁNDEZ ${ }^{1}$, SERGIO URIBE ${ }^{1,}$ SERGIO LAVANDERO ${ }^{3}$
}

\section{Myocardial lipids and creatine measured by magnetic resonance spectroscopy among patients with heart failure}

Background: Heart failure (HF) is characterized, among other features, by the development of alterations in myocardial energy metabolism, involving a decrease in glucose utilization and increased free fatty acid uptake by cardiomyocytes, associated with decreased deposits of high-energy phosphates (creatine phosphate/ creatine transporter). Magnetic resonance (MR) imaging allows a direct and noninvasive assessment of myocardial metabolites. Aim: To measure myocardial creatine and lipids by MR spectroscopy among patients with HF. Material and Methods: Cardiac MR spectroscopy (1.5 Tesla) with Hydrogen antenna and single voxel acquisition was performed in five patients with non-ischemic heart failure, aged $58 \pm 9.7$ years, (60\% males) and 5 healthy volunteers matched for age and sex. We analyzed the signals of creatine (Cr), lipids $(L)$ and water $(W)$ in the interventricular septum, establishing the water/lipid (W/L) and water/creatine (W/Cr) index to normalize the values obtained. Results: Among patients, left ventricular ejection fraction was $32 \pm 6.9 \%, 60 \%$ were in functional capacity II, 60\% had hypertension and one was diabetic. Spectroscopic curves showed a depletion of total Cr, evidenced by the W/ $C r$ index, among patients with heart failure, when compared with healthy controls $(1.46 \pm 1.21$ and $5.96 \pm 2.25$ respectively, $p<0,05)$. Differences in myocardial lipid content, measured as the W/L index, were not significant (5.06 \pm 2.66 and $1.80 \pm$ 1.62 respectively, $p=0.08$ ). Conclusions: Among patients with heart failure of nonischemic etiology, there is a depletion of creatine levels measured by MR spectroscopy.

(Rev Med Chile 2010; 138: 1475-1479).

Key words: Creatine; Heart failure; Magnetic resonance spectroscopy.

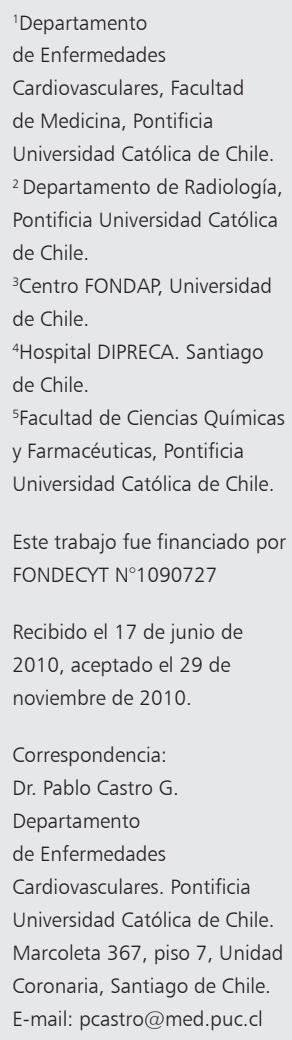

L a insuficiencia cardiaca (IC) es un problema de salud pública en aumento debido a la mayor expectativa de vida y la mayor presencia de comorbilidades ${ }^{1}$. Actualmente, existe creciente interés en el rol de la depleción energética como mecanismo asociado al desarrollo de esta patología. La célula miocárdica posee altos requerimientos energéticos, generando ATP principalmente en base a ácidos grasos y glucosa y posee un eficiente sistema de transporte de este 
producto. La principal vía por la cual se realiza este transporte es el de la creatin-kinasa (CK), la cual juega un rol importante en la movilización de fosfato desde la mitocondria a las miofibrillas mediante su acoplamiento con creatina. Durante el desarrollo de la IC se observa una disminución tanto de la actividad de la CK cardiaca como de los niveles de creatina y fosfocreatina. En el corazón del adulto, el mayor porcentaje del ATP se forma en base a lípidos como sustrato (60 a 90\%). El desarrollo progresivo de IC se acompaña de una disminución progresiva del uso de éstos como sustrato energético, con aumento proporcional del uso de glucosa. La acumulación de lípidos intracelulares produce toxicidad y mayor deterioro de la maquinaria energética² ${ }^{2}$.

El estudio de los componentes del metabolismo cardíaco requiere de muestras obtenidas de biopsias o de corazones al momento del trasplante. Recientemente, se ha descrito el uso de resonancia nuclear magnética (RNM) para investigar el metabolismo cardíaco in vivo, mediante la determinación de diferentes metabolitos en el tejido (por ejemplo, agua, creatina y lípidos). LA RNM tiene la ventaja de ser no invasiva, no emitir radiación y que, además de obtener información acerca de la estructura, función y perfusión, se puede obtener información completa de la interrelación fisiopatológica del corazón ${ }^{3}$.

El objetivo de este estudio es evaluar los niveles de creatina y lípidos mediante espectroscopia por resonancia nuclear magnética con antena de hidrógeno en pacientes con IC no isquémica.

\section{Métodos}

Se estudiaron 5 pacientes con IC y 5 controles sanos similares en edad y sexo. Los criterios de inclusión en el grupo de pacientes con IC, fueron: IC de etiología no isquémica determinada mediante cintigrama MIBI-dipiridamol o coronariografía, fracción de eyección de ventrículo izquierdo (FEVI) $<40 \%$ determinada por ventriculografía radio-isotópica, capacidad funcional 2 a 3 según NYHA, tratamiento médico convencional y estabilidad clínica en los últimos 4 meses. En todos los pacientes fueron registrados datos demográficos y se determinaron hemograma, creatinina, electrolitos plasmáticos y proBNP por los métodos de laboratorio habituales. El valor de proBNP normal se consideró $<125 \mathrm{pg} / \mathrm{mL}$. Se realizó espectrofotometría por RNM en los 5 pacientes y 5 controles sanos con antena de hidrógeno en un resonador de 1,5 Tesla, con técnica de adquisición de voxel único y sin gatillamiento cardiaco ni respiratorio. Se analizó una muestra de interés localizada en el septum interventricular de $8 \mathrm{~cm}^{3}$ y se obtuvo una curva espectroscópica con supresión parcial de la señal de agua. Se analizaron datos del área bajo la curva (AUC) de la señal de creatina $(3,0$ ppm) lípidos $(1,4 \mathrm{ppm})$ y agua $(4,8 \mathrm{ppm})$ como una medición de referencia. La señal de agua se utiliza para establecer una razón con lípidos (W/L) y creatina (W/Cr) para normalizar los valores obtenidos y compararlos entre sujetos ${ }^{4}$. Debido a la distribución no normal de los resultados, para analizar las variables continuas independientes se utilizó el test U de Mann-Whitney. Se consideró un valor p menor de 0,05 como estadísticamente significativo. Todos los pacientes y controles firmaron el consentimiento informado aprobado por el Comité de Ética de nuestra institución.

\section{Resultados}

Tres de los cinco pacientes eran de sexo masculino. La edad media de los pacientes fue $58 \pm$ 9,7 años. La etiología de la IC fue hipertensiva en 2 casos e idiopática en los restantes 3 (60\%) pacientes. Tres pacientes $(60 \%)$ presentaban una capacidad funcional 2 al momento del examen. La FEVI estimada por ventriculografía radioisotópica fue de $32 \pm 7 \%$. En general, los pacientes presentaban un volumen ventricular aumentado así como valores elevados de proBNP (Tabla 1). La Figura 1 muestra la imagen espectroscópica de un paciente con IC (A) y un control sano (B). En ella se pueden observar en las integrales obtenidas de agua, creatina y lípidos que permiten estimar los índices W/Cr y W/L. No hubo diferencias entre pacientes y controles en cuanto a sus características demográficas basales.

El índice W/Cr de los pacientes con IC fue significativamente mayor, comparado al de los controles $(5,96 \pm 2,25$ vs $1,46 \pm 1,21 ; \mathrm{p}<0,05)$, lo que refleja un menor contenido de creatina en el miocardio de los individuos con IC. Con respecto al índice $\mathrm{W} / \mathrm{L}$, se observó una tendencia no significativa a un menor valor en los pacientes con IC comparado a los controles sanos $(1,80 \pm 1,62$ 
Niveles de creatina y lípidos en miocardio - J.L. Winter et al

Tabla 1. Características de los pacientes

\begin{tabular}{|ll|}
\hline Edad & $58 \pm 9,7$ años \\
\hline Sexo masculino & 3 pacientes \\
\hline Peso & $69 \pm 11,7 \mathrm{Kg}$ \\
\hline Capacidad funcional II/III & $3 / 2$ pacientes \\
\hline VFDVI & $252 \pm 70 \mathrm{ml}$ \\
\hline Fracción eyección VI & $32 \pm 6,9 \%$ \\
\hline Pro-BNP & $1.279 \pm 948 \mathrm{pg} / \mathrm{ml}$ \\
\hline HTA & 3 paciente \\
\hline DM & 1 paciente \\
\hline
\end{tabular}

vs $5,06 \pm 2,66$, pacientes vs controles, respectivamente; valor- $\mathrm{p}=0,08)$. Los resultados se grafican en la Figura 2.

\section{Discusión}

En este estudio, se demuestra que los pacientes con miocardiopatia dilatada de origen no isquémico presentan una disminución de los niveles de creatina y aumento de los niveles de lípidos al ser comparados con controles sanos.

La creatina es parte fundamental de la cadena

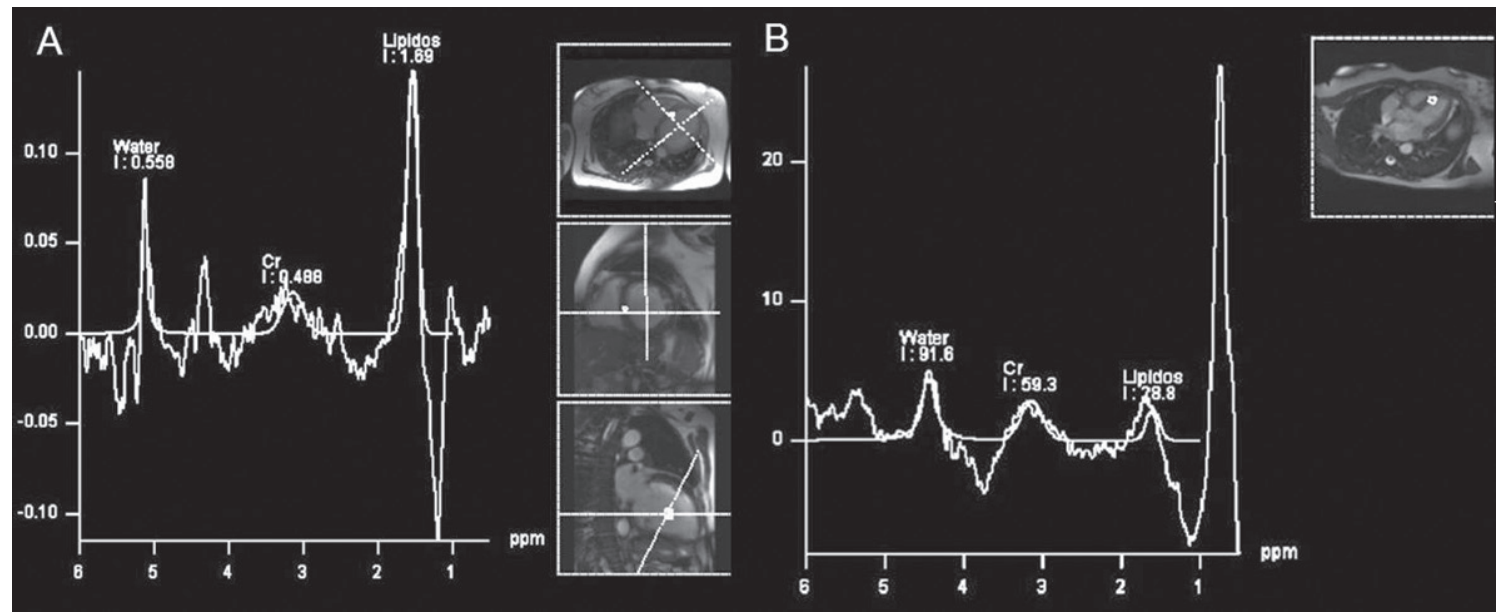

Figura 1. Espectroscopia con antena de hidrógeno de una paciente de 55 años con insuficiencia cardiaca (A) y una mujer control de 49 años (B). Se puede observar la diferencias en las concentraciones de agua, creatina y lípidos entre ambos sujetos.

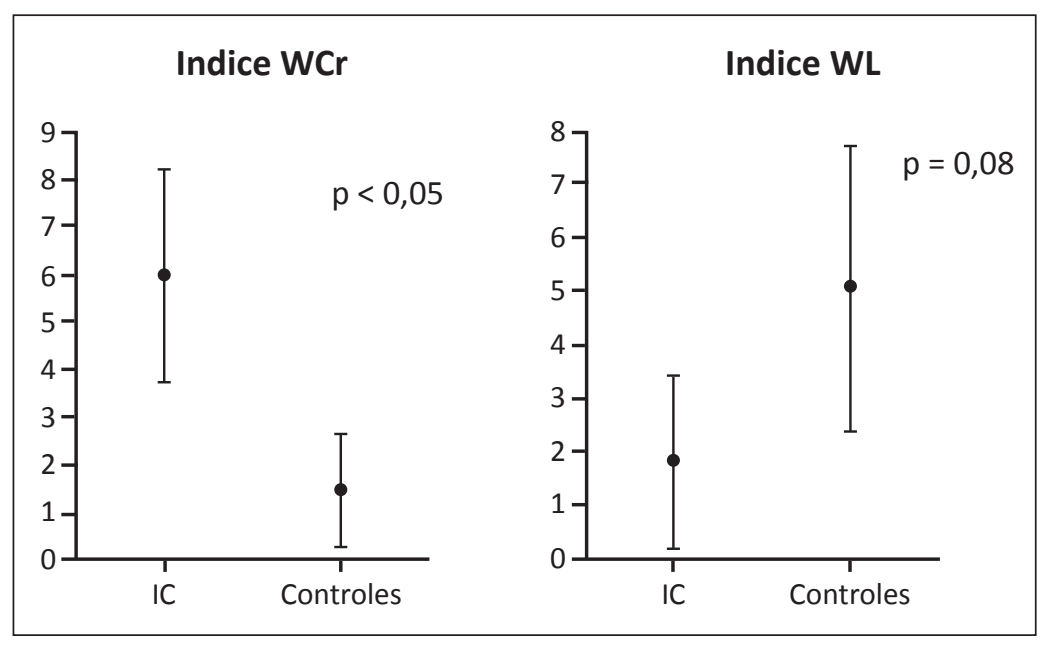

Figura 2. Box plot que muestra los índices agua/creatina $(\mathrm{W} / \mathrm{Cr})$ y agua/lípidos (W/L) en pacientes con insuficiencia cardiaca e individuos sanos. Se observa un aumento estadísticamente significativo en el índice W/Cry una tendencia no significativa a la disminución del índice W/L en los sujetos con insuficiencia cardiaca. 
de transporte de energía desde las mitocondrias del cardiomiocito hacia las miofibrillas. El alto requerimiento energético de estas fibras requiere de una rápida disponibilidad de ATP para su funcionamiento, siendo el sistema de la creatinkinasa (CK) la que permite el acoplamiento de fosfato a la creatina a nivel mitocondrial para su posterior desacoplamiento y formación de ATP en las miofibrillas 5 .

En la IC se ha demostrado alteraciones en múltiples componentes del sistema de la CK Primero, existe una disminución de los niveles de creatina y actividad de la CK. Lo anterior ha sido estudiado en biopsias cardiacas en pacientes con miocardiopatía dilatada idiopática donde se observó una disminución de $51 \%$ en los niveles de Cr y $34 \%$ en la actividad de $\mathrm{CK}$ al compararlos con corazones de donantes ${ }^{6}$. Uno de los mecanismos que explican su disminución es la baja disponibilidad (downregulation) del cotransportador sodio-creatina ( $\mathrm{Na}-\mathrm{Cr}$ ), el cual es responsable del ingreso de $\mathrm{Cr}$ al miocito ${ }^{7}$. Por otra parte, un estudio reciente demostró en pacientes con miocardiopatía dilatada no isquémica, una disminución del índice fosfocreatina (PCr)/ATP determinado mediante el uso de antenas de fosforo-31 con medición de los niveles de fosfocreatina y ATP ${ }^{8}$. Del mismo modo, se ha reportado que en los pacientes portadores de miocardiopatía hipertrófica presentan una depleción de $\mathrm{Cr}$ en comparación con individuos sanos pero sus niveles se encuentran por sobre los pacientes con miocardiopatía dilatada ${ }^{4}$.

Por otro lado, en la IC el sustrato energético principal son los ácidos grasos, siendo menor la contribución de otras sustancias como glucosa, aminoácidos y lactato. Sin embargo, el metabolismo de los ácidos grasos puede ser alterado por múltiples factores. En la IC se ha demostrado un desplazamiento del metabolismo de ácidos grasos por el uso de glucosa. En un estudio realizado mediante la medición de $\mathrm{Cr}$ con espectroscopia con antena de hidrogeno y el uso de cintigrafía con análogos radioactivos de ácidos grasos (123I BMI$\mathrm{PP}$ ) en pacientes con diversas patologías cardiacas mostró una disminución del índice de captación miocárdica de ácidos grasos versus mediastino $(\mathrm{H} / \mathrm{M})$, el cual se correlaciona directamente con la concentración miocárdica de Cr y la función sistólica? .

La espectroscopia por resonancia magnética cardiaca permite la determinación in vivo de metabolitos en el miocardio. Existen múltiples antenas para la detección del los metabolitos en el tejido: hidrógeno, fosforo, carbono y sodio; siendo la de hidrógeno la más utilizada clínicamente. La espectroscopia con antena de fósforo permite la determinación de P-Cr, ATP y fosfato inorgánico ${ }^{10}$, pero su sensibilidad está determinada a ciertas regiones del miocardio, especialmente la pared anterior del ventrículo izquierdo ${ }^{11}$. Recientemente, se ha descrito el empleo combinado de antenas de hidrógeno y fósforo, sin embargo, su técnica de adquisición es compleja, requiriendo hasta 2 horas en la obtención de las imágenes ${ }^{4}$. Por otra parte, la antena de hidrógeno ha demostrado tener una mayor sensibilidad que la antena de fósforo con una especial capacidad de detectar el pool total de $\mathrm{Cr}$ (tanto fosforilada como no fosforilada) ${ }^{11}$. La antena de hidrógeno permite la detección de múltiples metabolitos como: agua (4,7 ppm), creatina (3,0 ppm), lípidos (1,4 ppm), lactato (1,3 ppm), etc. La intensidad y ancho de las curvas inscritas en cada una de estas posiciones definen la cantidad de estos metabolitos en el tejido examinado. Estudios en animales demuestran en que existe una excelente correlación entre la cantidad de metabolitos detectados por esta técnica y la realizada a través del estudio bioquímico del tejido ${ }^{12}$.

Nuestros resultados son similares a los publicados por Nakae y cols, quienes determinaron los niveles de creatina en 11 pacientes con miocardiopatía dilatada mediante el uso de espectroscopia con antena de hidrógeno ${ }^{4}$. En este estudio, se observó una disminución significativa de los niveles de creatina en los pacientes con miocardiopatía dilatada (16 vs $28 \mu \mathrm{mol} / \mathrm{g}$ de peso seco en comparación con sujetos sanos), además, se estableció una correlación positiva de los niveles de creatina con la FEVI y una relación inversa con los niveles de BNP. La espectroscopia por RNM también ha demostrado ser de utilidad para evaluar viabilidad miocárdica. Bottomley y cols realizaron un examen de espectroscopia a 10 pacientes con el antecedente de infarto cardíaco, observando una disminución estadísticamente significativa de los niveles de creatina en las zonas infartadas en comparación con las no infartadas y las de individuos sanos ${ }^{11}$.

Este trabajo presenta como limitaciones el pequeño número de pacientes, dificultando lograr significancia estadística y, por otra parte, la espectroscopia por RNM es un examen no ampliamente 
disponible, con tiempos largos de adquisición de imágenes y que requieren operadores entrenados para su interpretación.

Nuestros resultados, sugieren que en pacientes portadores de miocardiopatía dilatada de etiología no isquémica, existe una depleción de los niveles de creatina medidos mediante el uso de espectroscopia por resonancia nuclear magnética. El uso de este examen se presenta como una alternativa no invasiva promisoria para la evaluación del metabolismo cardíaco.

\section{Referencias}

1. Gómez-Soto FM, Andrey JL, García-Egido AA, Escobar MA, Romero SP, García-Arjona R, et al. Incidence and mortality of heart failure: A community-based study. Int J Cardiol 2010 May 13.

2. Neubauer S. The failing heart-an engine out of fuel. $\mathrm{N}$ Engl J Med 2007 March 15; 356: 1140-51.

3. Hudsmith LE, Neubauer S. Magnetic resonance spectroscopy in myocardial disease. JACC Cardiovasc Imaging 2009; 2: 87-96.

4. Nakae I, Mitsunami K, Omura T, Yabe T, Tsutamoto T, Matsuo S, et al. Proton magnetic resonance spectroscopy can detect creatine depletion associated with the progression of heart failure in cardiomyopathy. J Am Coll Cardiol 2003; 42: 1587-93.

5. Bittl JA, Ingwall JS. Reaction rates of creatine kinase and ATP synthesis in the isolated rat heart. A 31P NMR magnetization transfer study. J Biol Chem 1985; 260: 3512-7.

6. Nascimben L, Ingwall JS, Pauletto P, Friedrich J, Gwathmey JK, Saks V, et al. Creatine kinase system in failing and nonfailing human myocardium. Circulation 1996; 94: 1894-901.

7. Neubauer S, Remkes H, Spindler M, Horn M, Wiesmann F, Prestle J, et al. Downregulation of the $\mathrm{Na}(+)$-creatine cotransporter in failing human myocardium and in experimental heart failure. Circulation 1999; 100: 1847-50.

8. Chida K, Otani H, Kohzuki M, Saito H, Kagaya Y, Takai $\mathrm{Y}$, et al. The relationship between plasma BNP level and the myocardial phosphocreatine/adenosine triphosphate ratio determined by phosphorus-31 magnetic resonance spectroscopy in patients with dilated cardiomyopathy. Cardiology 2006; 106: 132-6.

9. Nakae I, Mitsunami K, Matsuo S, Horie M. Creatine depletion and altered fatty acid metabolism in diseased human hearts: clinical investigation using $1 \mathrm{H}$ magnetic resonance spectroscopy and 123I BMIPP myocardial scintigraphy. Acta Radiol 2007; 48: 436-43.

10. Raman SV, Simonetti OP. The CMR examination in heart failure. Heart Fail Clin 2009; 5: 283-300, v.

11. Bottomley PA, Lee Y, Weiss RG. Total creatine in muscle: imaging and quantification with proton MR spectroscopy. Radiology 1997; 204: 403-10.

12. Szczepaniak LS, Dobbins RL, Metzger GJ, SartoniD'Ambrosia G, Arbique D, Vongpatanasin W, et al. Myocardial triglycerides and systolic function in humans: in vivo evaluation by localized proton spectroscopy and cardiac imaging. Magn Reson Med 2003; 49: 417-23. 